\title{
Hypercalcemia in metastatic breast cancer unrelated to skeletal metastasis
}

\author{
Heather Sigvaldason MPAS BMR(PT), Adebola Obayan MD PhD, Ken von Kuster MD, K. Alok Pathak MD
}

1 48-year-old woman was given a diagnosis of infiltrating ductal breast carcinoma (T2N1M0; grade I; positive for estrogen receptors, progesterone receptors and human epidermal receptor-2 [HER2] status; with 3/31 positive nodes). Treatment at that time involved left lumpectomy with axillary node dissection followed by chemotherapy and radiotherapy. Thirteen years later, she was given a diagnosis of breast cancer recurrence with liver metastasis that was positive for estrogen receptors and HER2 status, and negative for progesterone receptors, based on a core biopsy. Other than the liver metastasis, computed tomography of the brain, chest, abdomen and pelvis showed no other site of distant metastasis, including the visualized skeletal system. A bone scan showed several foci of abnormal radiotracer accumulation consistent with degenerative changes as opposed to metastatic disease. Treatment was initiated, including capecitabine, trastuzumab and pamidronate.

The patient had hypercalcemia documented on multiple occasions, with corrected calcium levels ranging from 2.72 to 2.85 (normal 2.1-2.6) $\mathrm{mmol} / \mathrm{L}$, dating back to the time of diagnosis of her breast cancer. She did not have symptoms directly attributable to hypercalcemia apart from one episode of nephrolithiasis 11 years after diagnosis. Hypercalcemia was again noted two years later, with a serum corrected calcium level of $2.95 \mathrm{mmol} / \mathrm{L}$.

The patient's serum parathyroid hormone was elevated at 276 (normal 7-50) ng/L. There were no palpable abnormalities in the thyroid or neck on physical examination. Ultrasonography revealed a $3-\mathrm{cm}$ mass posterior to the lower pole of the right lobe of the thyroid (Figure 1). Parathyroid scan identified a lesion at the posterior inferior aspect of the right thyroid, showing excess sestamibi accumulation and slow washout, consistent with parathyroid adenoma (Figure 2). Despite multiple treatments with pamidronate, hypercalcemia persisted, with levels of serum corrected calcium around $2.6 \mathrm{mmol} / \mathrm{L}$.
She was referred for consideration of surgical management of primary hyperparathyroidism.

The patient underwent a minimally invasive right inferior parathyroidectomy for management of primary hyperparathyroidism. Her parathyroid hormone level dropped to $41 \mathrm{ng} / \mathrm{L}$ in the immediate postoperative period. Postoperative recovery was uneventful, and a normal serum corrected calcium level of $2.2 \mathrm{mmol} / \mathrm{L}$ was observed five weeks after surgery. Her corrected calcium level remains normal at $2.2 \mathrm{mmol} / \mathrm{L}$, more than two years after surgery.

Histopathologic examination confirmed a 6-g well-circumscribed adenoma measuring $2.7 \times 1.7$ $\times 2.0 \mathrm{~cm}$. A deposit of metastatic breast carcinoma was incidentally identified within the adenoma (Figure 3A). Immunohistochemical stains for parathyroid hormone were positive in the adenoma (Figure 3B) and negative within the second atypical cell population, which showed strong positive staining for cytokeratin 7 (Figure 3C), estrogen receptors (Figure 3D) and progesterone receptors but was negative for thyroid transcription factor 1 , thyroglobulin, cytokeratin 20 and caudal type homeobox. The atypical cells were strongly positive for HER2/neu (3+) with more than $50 \%$ cells showing intense membrane staining.

This patient is presently taking trastuzumab, tamoxifen and dexamethasone for liver and lung metastases. As well, she is receiving stereotactic radiotherapy for newly diagnosed cerebral metastasis.
Competing interests: None declared.

This article has been peer reviewed.

The authors have obtained patient consent.

Correspondence to:

K. Alok Pathak, apathak@ cancercare.mb.ca

CMAJ 2016. DOI:10.1503 /cmaj.150638 


\section{Discussion}

Hypercalcemia has been reported in as many as $30 \%-40 \%$ of patients with breast cancer. ${ }^{1}$ Although the incidence is likely to be lower now, with the current systemic therapies and widespread use of bisphosphonates, breast cancer remains one of the most common cancers associated with hypercalcemia. Although bony metastasis is by far the most common cause of hypercalcemia in patients with breast cancer (20\%-30\%), the differential diagnosis also includes primary hyperparathyroidism and, less commonly, humoral hypercalcemia of malignancy. ${ }^{2}$ Primary hyperparathyroidism is the most common cause of benign hypercalcemia and affects about $1 \%$ of adults, mostly postmenopausal women. ${ }^{3}$

According to the guidelines from the National Comprehensive Cancer Network, ${ }^{4}$ hypercalcemia may be an indicator of breast cancer progression, and a bone scan is recommended to detect suspected skeletal metastasis. If there is no evidence of osseous involvement, other alternative causes should be sought. Serum parathyroid hormone provides a simple way to differentiate between hypercalcemia caused by primary
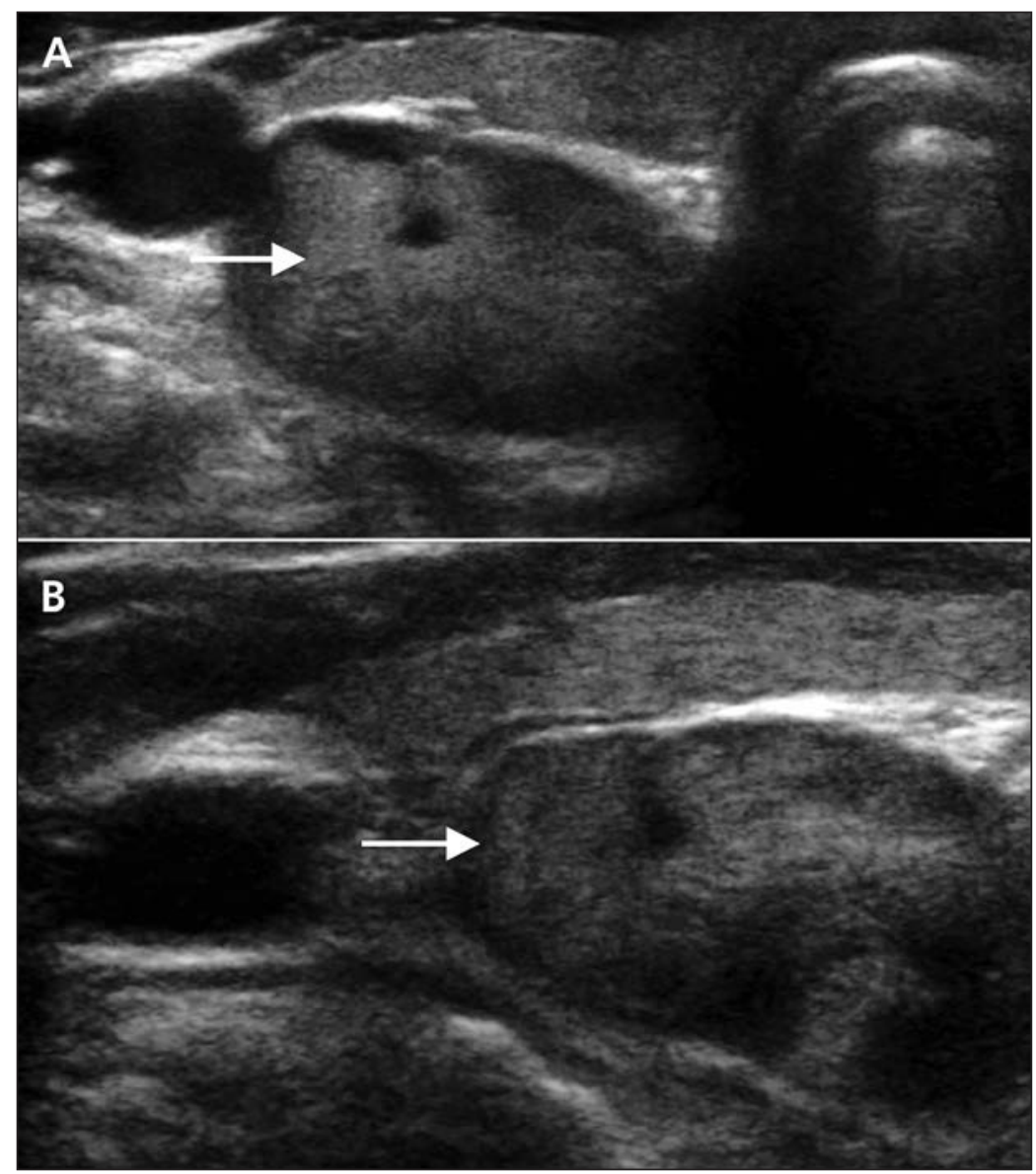

Figure 1: Ultrasonographic image of the neck of a 48-year-old woman showing transverse (A) and sagittal (B) views of the parathyroid adenoma. hyperparathyroidism and other causes. Parathyroid hormone levels would be normal or elevated in the setting of primary hyperparathyroidism but suppressed with bony metastasis or humoral hypercalcemia of malignancy. ${ }^{5}$ If the level of parathyroid hormone is inappropriately elevated, a parathyroid scan (Figure 2) should be performed to assess for an adenoma as well as ultrasonography (Figure 1) for anatomic localization.

In appropriately selected patients, surgery is the recommended management for primary hyperparathyroidism, with excellent results. ${ }^{2}$ For a single adenoma, minimally invasive parathyroidectomy is recommended, whereas bilateral cervical exploration may be required for multigland involvement or failed localization.

Multiple studies have assessed the relation between primary hyperparathyroidism and malignancy. The largest of these, by Nilsson and colleagues, ${ }^{3}$ provided a large retrospective analysis of patients with prior parathyroidectomies for primary hyperparathyroidism. The authors reported an overall increase in frequency of all types of cancer in both sexes, with the largest proportion being breast cancer in women. The frequency of breast cancer was significantly increased relative to a control population (hazard ratio $1.44,95 \%$ confidence interval $1.25-1.62){ }^{3}$ In a retrospective review by Tanaka, ${ }^{2}$ primary hyperparathyroidism was reported in $2.88 \%$ of patients with breast cancer, compared with $0.04 \%-0.08 \%$ in the adult female population. A case-control study involving 100 patients found a $7 \%$ incidence of primary hyperparathyroidism in patients with treated breast cancers (v. $0 \%$ in the control group of patients with thyroid tumours). ${ }^{6}$

The reason for this relation is not known, but various theories have been proposed. The increased risk of malignancy was found to persist even 15 years after parathyroidectomy, suggesting genetic predisposition or environmental

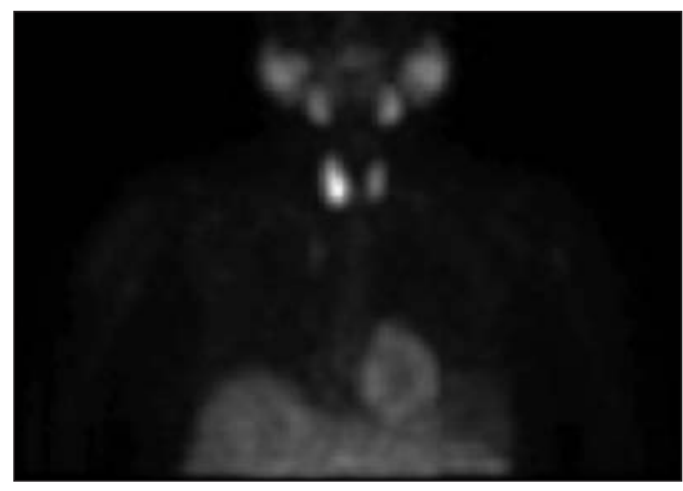

Figure 2: Dual isotope parathyroid scan with technetium $99 \mathrm{~m}$ pertechnetate $(53 \mathrm{MBq})$ and technetium 99m sestamibi (992 MBq) showing excess sestamibi accumulation and slow washout, consistent with parathyroid adenoma. 
factors as causal mechanisms, rather than biochemical changes. ${ }^{3}$ The close follow-up of patients with hypercalcemia could be a confounding factor for this observed association. A large observational study involving a cohort of such patients who do not undergo parathyroidectomy could potentially answer this question.

\section{Tumour-to-tumour metastasis}

This case involved an unexpected finding of metastatic breast cancer within the parathyroid adenoma. Based on criteria presented by Campbell in $1968,{ }^{7}$ this would be considered a true case of tumour-to-tumour metastasis. The following four criteria are required to qualify: more than one primary tumour is present, the recipient tumour is a true neoplasm, the metastatic neoplasm is a true metastasis (i.e., showing established growth in the recipient tumour and not resulting from contiguous growth or embolization of tumour cells), and lymphatic systems as well as lymphoreticular malignant tumours are excluded. ${ }^{7}$ This phenomenon is a rare event, with only about 100 cases reported in the English literature. ${ }^{8}$

The primary tumour is typically more aggressive than the recipient tumour, with lung cancer being the most frequent primary tumour, fol- lowed by breast and prostate. Renal cell cancer is the most frequent malignant recipient, and meningioma is the most frequent benign recipient. ${ }^{5}$ Of note, endocrine tumours, including parathyroid tumours, were found to comprise $45 \%$ of recipient lesions, possibly because the high vascularity of endocrine tissue provides a good recipient bed for metastasis. ${ }^{9}$

Tumour-to-tumour metastasis involving the parathyroid glands is considered rare; however, its true incidence is largely unknown, perhaps because parathyroid glands are not routinely assessed during autopsy. Autopsy studies involving patients with known cancer by Gattuso and colleagues ${ }^{10}$ reported an incidence of parathyroid metastasis between $0.2 \%$ and $11.9 \%$. The most common sites of origin, in decreasing order of incidence, were breast, blood (leukemia), skin (malignant melanoma), lung, soft tissue (spindle cell sarcoma) and lymph nodes (lymphoma). ${ }^{10}$

There have been few reported cases of breast cancer metastasizing to a parathyroid gland. ${ }^{5,8,11}$ Parathyroid adenomas were the recipients in three previously reported cases, and one case of four-gland hyperplasia with metastatic disease involving one of the hyperplastic glands was observed. ${ }^{5,8,11}$ In two of these cases, the parathyroid involvement was the only evidence of dis-

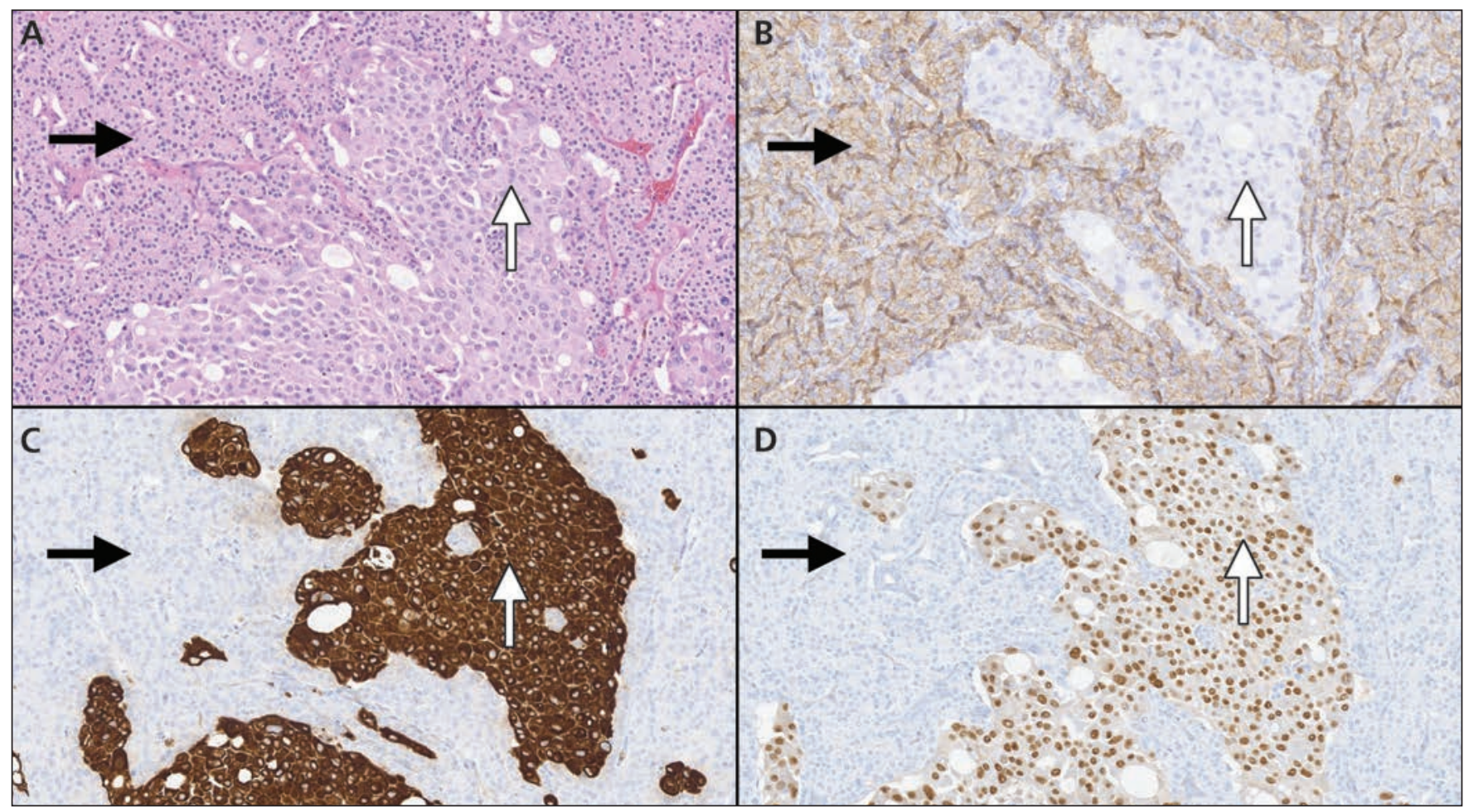

Figure 3: Histopathologic sections showing two distinct cell populations with larger breast carcinoma cells (white arrows) arranged as irregular solid clusters with focal gland formation and nuclear atypia, and smaller parathyroid cells (black arrows) arranged as cords and nests with bland nuclear features in hematoxylin-eosin stain (A). The parathyroid tissue shows strong positive staining for parathyroid hormone (B) and is negative for cytokeratin-7 (C) and estrogen receptors (D). The breast carcinoma cells show strong positive staining for cytokeratin-7 (C) and estrogen receptors (D) and are negative for parathyroid hormone (B). (Taken with a $\times 10$ objective lens, with total magnification $\times 100$.) 
seminated disease, with the remaining showing widespread disease. . $^{5,8,11}$

The current case illustrates the importance of considering a second unrelated pathology in the setting of hypercalcemia in a patient with breast cancer. Hypercalcemia is a common complication of breast cancer, and primary hyperparathyroidism is one of the potential causes. Proper evaluation is warranted to rule out the presence of parathyroid adenoma, as this is a surgically curable condition. In contrast, hypercalcemia related to occult distant metastatic disease is associated with a very poor prognosis.

Minimally invasive parathyroidectomy did not alter the metastatic course of this patient's breast cancer, but it was successful in long-term control of her hypercalcemia without the use of bisphosphonate therapy and its associated risks. This case also describes a rare occurrence of tumour-to-tumour metastasis involving the parathyroid glands.

\section{References}

1. Michels KB, Xue F, Brandt L, et al. Hyperparathyroidism and subsequent incidence of breast cancer. Int J Cancer 2004;110:449-51.

2. Tanaka Y. Primary hyperparathyroidism with breast carcinoma. Breast Cancer 2010;17:265-8.

3. Nilsson IL, Zedenius J, Yin L, et al. The association between primary hyperparathyroidism and malignancy: nationwide cohort analysis on cancer incidence after parathyroidectomy. Endocr Relat Cancer 2007; 14:135-40.

4. NCCN Clinical Practice Guidelines in Oncology (NCCN Guidelines). Breast cancer. Version 3.2015. Fort Washington (PA): National Comprehensive Cancer Network; 2015.

5. Lee SH, Kim BH, Bae MJ, et al. Concurrence of primary hyperparathyroidism and metastatic breast carcinoma affected a parathyroid gland. J Clin Endocrinol Metab 2013;98:3127-30.

6. Fierabracci P, Pinchera A, Miccoli P, et al. Increased prevalence of primary hyperparathyroidism in treated breast cancer. $J$ Endocrinol Invest 2001;24:315-20.

7. Campbell LV Jr, Gilbert E, Chamberlain CR Jr, et al. Metastases of cancer to cancer. Cancer 1968;22:635-43.

8. Lee HE, Kim DH, Cho YH, et al. Tumor-to-tumor metastasis: hepatocellular carcinoma metastatic to parathyroid adenoma. Pathol Int 2011;61:593-7.

9. Shifrin A, LiVolsi V, Shifrin-Douglas S, et al. Primary and metastatic parathyroid malignancies: A rare or underdiagnosed condition? J Clin Endocrinol Metab 2015;100:E478-81.

10. Gattuso P, Khan NA, Jablokow VR, et al. Neoplasms metastatic to parathyroid glands. South Med J 1988;81:1467.

11. Woolner LB, Keating FR Jr, Black BM. Primary hyperparathyroidism and metastatic breast carcinoma: a case in which breast carcinoma metastasized to a parathyroid adenoma. Cancer 1958 11:975-9.

Affiliations: Section of Surgical Oncology, CancerCare Manitoba; Departments of Surgery (Sigvaldason, Obayan, Pathak) and Pathology (VonKuster), University of Manitoba, Winnipeg, Man.

Contributors: All of the authors wrote and revised the article, gave final approval of the version to be published and agreed to act as guarantors of the work.

The section Cases presents brief case reports that convey clear, practical lessons. Preference is given to common presentations of important rare conditions, and important unusual presentations of common problems. Articles start with a case presentation (500 words maximum), and a discussion of the underlying condition follows (1000 words maximum). Visual elements (e.g., tables of the differential diagnosis, clinical features or diagnostic approach) are encouraged. Consent from patients for publication of their story is a necessity. See information for authors at www.cmaj.ca. 